\title{
Las prácticas pedagógicas de los docentes en Colombia, en función del Proyecto Educativo Institucional (PEI)
}

The pedagogical practices of teachers throughout the Colombian territory are due to the Institutional Educational Project

As práticas pedagógicas dos professores na Colômbia, com base no Projeto Educacional Institucional (PEI)

Luz Migdonia Gómez Guzmán1

luzmigogu@gmail.com

\section{Sunny Raquel Perozo Chirinos ${ }^{2}$}

sunperozo@gmail.com

2Universidad Metropolitana de Educación, Ciencia y Tecnología, Panamá

${ }^{2}$ Universidad del Zulia Núcleo Costa Oriental del Lago, Venezuela

Recibido diciembre 2019| Revisado enero 2020 | Publicado 01 de abril 2020

\section{RESUMEN}

El propósito de este artículo es presentar un abordaje teórico y conceptual sobre cómo las prácticas pedagógicas de los docentes de Colombia, desarrolladas a partir de lo dispuesto en el Proyecto Educativo Institucional (PEI) de cada escuela o colegio, contempladas como un instrumento identificador y propio de cada institución para orientar el logro de la calidad educativa planteada en la nueva constitución política de Colombia del año 1991. Metodológicamente se planteó un acercamiento teórico y crítico utilizando dos niveles, monográfico dirigido a la revisión de las fuentes para recopilar las ideas de los planteamientos conceptuales y luego se desarrolló un nivel hermenéutico en el cual se hizo una interpretación a partir de la sustentación, vigencia y alcance. La reflexión final de este análisis teórico conlleva a confirmar la importancia de la integración de lo dispuesto en el proyecto PEI en las prácticas educativas, para lo que se hace necesario que el docente esté plenamente identificado con sus fines y objetivos.

Palabras clave: Proyecto educativo institucional; calidad; autonomía; identidad; institución, prácticas pedagógicas

\section{ABSTRACT}

The purpose of this article is to present a theoretical and conceptual approach on how the pedagogical practices of teachers in Colombia, developed from the provisions of the Institutional Educational Project (PEI) of each school or college, considered as an identifying and proper instrument of each institution to guide the achievement of educational quality raised in the new Colombian political constitution of 1991. Methodologically, a theoretical and critical approach was proposed using two levels, monographic aimed at reviewing the sources to collect the ideas of the approaches conceptual and then a hermeneutic level was developed in which an interpretation was made based on the support, validity and scope. The final reflection of this theoretical analysis leads to confirming the importance of integrating the provisions of the PEI project into educational practices, for which it is necessary that the teacher is fully identified with its aims and objectives.

Key words: Institutional educational project; quality; autonomy; identity; institution, pedagogical practices 


\section{RESUMO}

O objetivo deste artigo é apresentar uma abordagem teórica e conceitual de como as práticas pedagógicas dos professores na Colômbia se desenvolveram a partir das disposições do Projeto Educacional Institucional (PEI) de cada escola ou faculdade, considerado um instrumento de identificação e adequado de cada instituição para orientar a obtenção da qualidade educacional levantada na nova constituição política colombiana de 1991. Metodologicamente, uma abordagem teórica e crítica foi proposta usando dois níveis, monográficos, com o objetivo de revisar as fontes para coletar as ideias das abordagens. Conceitual e, em seguida, foi desenvolvido um nível hermenêutico, no qual foi feita uma interpretação com base no suporte, validade e escopo. A reflexão final dessa análise teórica leva a confirmar a importância de integrar as disposições do projeto PEI nas práticas educacionais, para as quais é necessário que o professor seja totalmente identificado com suas metas e objetivos.

Palavras-chave: Projeto educacional institucional; qualidade; autonomia; identidade; instituição, práticas pedagógicas

\section{INTRODUCCIÓN}

La educación como derecho congénito de todo hombre y mujer en el mundo, es un proceso importante y trascendental para las personas y el desarrollo social de los países, lo que la convierte en un motor de transformación y avance de los pueblos y al mismo tiempo plantea la necesidad de una constante revisión, análisis y evaluación de cada uno de sus componentes y actores del proceso educativo: docentes, recursos, estrategias, modelos y propuestas con el fin de cumplir con su cometido social, cultural y de formación humana a través del mejoramiento de todos y cada uno de ellos.

En este sentido, Araya (2007) se refiere a la educación como un proceso orientado a lograr la transmisión de normas de conducta, modelos de ser y formas de ver el mundo a las nuevas generaciones. Además, este autor expresa que el proceso educativo es un componente de la socialización de las personas, lo cual le da un carácter activo, es decir cada generación aporta su modo de entender el mundo, por lo cual es importante que desde la escuela se le dé a la educación un carácter dinámico y protagónico en procesos de transformación y de convivencia en cada región.

En este orden de ideas, la educación es un proceso importante en cada país, territorio, contexto. Schleicher (2016) recuerda que ésta ha sido a través de los años un pilar para toda sociedad, que de ella se han encargado con esmero personas, entidades, gobiernos, entre otros, debido a su importancia $y$ trascendencia, de ahí que se entienda el afán y las continuas propuestas por su mejoramiento, a través de los años, por parte de los gobiernos de los diferentes países del mundo.

Al respecto la UNESCO (2017) afirma en uno de sus informes que los países latinoamericanos con altos ingresos del 2008 al 2014, el $84 \%$ de los jóvenes terminaron el segundo ciclo de la enseñanza en comparación con un $43 \%$ en países de ingresos medios altos y un $38 \%$ en los países de ingresos medios bajos y más preocupante, solo un $14 \%$ en los de bajos ingresos.

Las exigencias y los retos se acrecientan y con ellos las propuestas y los nuevos proyectos, de allí que por razones obvias (la falta de calidad educativa, la deserción, la repitencia, entre otras) tanto los gobiernos como las organizaciones internacionales: OCDE, UNESCO, Banco de la República mantienen a la educación, en los últimos años, como agenda permanente.

Pero, no será la educación tema de solo grandes organizaciones, también lo es de cada 
quien: padres de familia, directivos, docentes y sociedad en general que ven en ésta la estrategia de mejoramiento global; hacer caso o no a estas propuestas y cambiar de actitud a manera personal y profesional ya no es solo una posible opción, es un deber y la obligación de todos, en especial los que directamente están responsabilizados de la transformación de las personas y con ellas de las naciones enteras.

Se dice con cierta sabiduría que la educación es la encargada del desarrollo y el avance de las sociedades, y si esto no se da como tal, es porque se ha quedado en palabras sin que docentes, familias y la comunidad educativa en general se den a la tarea de hacer que esto suceda.

En aras a toda esta realidad, existen movimientos como el de "Educación para todos" (EPT) de la UNESCO que procura a nivel mundial proporcionar educación básica de calidad a todos los niños, jóvenes y adultos" (UNESCO 2000). Acciones como éstas gestan oportunidades a poblaciones enteras que tienen altos índices de personas en condición de analfabetismo, niños desescolarizados y por ende auguran futuros poco promisorios sin oportunidades de progreso y empleo en su vida adulta.

Estos flagelos ponen en la mira a la educación a nivel mundial mediante retos como buscar que se promulgue la equidad y que se cumpla el legado del derecho a la educación de calidad para todos los ciudadanos.

Todo este esfuerzo de quién genera luchas en todo el continente (pobreza, desigualdad, falta de formación a docentes) procura desde la educación que se atiendan necesidades como el logro de la paz, el desarrollo sostenible y el diálogo intercultural, con la finalidad de que no existan barreras ni brechas en los intercambios sociales, que haya un avance conjunto entre las diferentes regiones y que se aumenten las oportunidades y posibilidades en el logro de los sueños de las personas.

\section{METODOLOGÍA}

Este trabajo se orientó a presentar un abordaje teórico y conceptual sobre cómo las prácticas pedagógicas de los docentes de Colombia se desarrollan, en cada escuela o colegio, a partir de lo dispuesto en el Proyecto Educativo Institucional (PEI), contemplado éste por las políticas nacionales de educación como un instrumento identificador, propio de cada institución para orientar el logro de la calidad educativa planteada en la nueva constitución política de Colombia del año 1991.

Para el proceso de documentación según lo dispuesto por Hurtado (2010), se utilizó los siguientes niveles:

- Nivel monográfico: En este nivel mediante la técnica de Riaceer la cual comprende la recopilación, identificación y almacenamiento, se revisaron los diferentes aspectos conceptuales planteados por los autores sobre los elementos contentivos en el PEI como son: la autonomía, la identidad que éste otorga a cada institución, la calidad educativa, así como su implementación y su realidad en el quehacer práctico en las instituciones.

- Nivel hermenéutico: Se realiza una interpretación de los aspectos conceptuales presentados en el nivel monográfico a partir del entendimiento, sustentación, alcance y vigencia de los planteamientos. 


\section{Fundamentación teórica}

Uribe (2006), aporta al tema de mejoramiento y transformación con datos puntuales que ratifican la dinámica educativa colombiana en su curso hacia el mejoramiento de los últimos años, al expresar que las transformaciones educativas en Colombia ocurrieron en la década de los cincuenta, cuando se presentó un rápido y sostenido crecimiento económico y un cambio significativo en la estructura económica y demográfica del país.

En efecto, el tema de calidad $y$ mejoramiento de la institucionalidad en Colombia, ha sido una lucha constante en cada mandato gubernamental donde algunos gobiernos (unos más enfáticos que otros), han dejado su huella en el proceso educativo colombiano.

Los principales cambios educativos en Colombia llegaron con el gobierno de López Pumarejo (1934-1938), quien consideró a la educación como una de las preocupaciones centrales. En este período de gobierno se culpó a la descentralización educativa como una de las principales causas del atraso de la educación en el país.

Se dice también que en el mandato del presidente Barco (1986 - 1990) se dieron procesos de descentralización y se inició la propuesta de mayor inversión y aumento en la partida hacia la autonomía política y administrativa de los entes territoriales. Con la ley 12 de 1986, se aumentaron las transferencias a los municipios, pero también aumentaron las funciones $y$ responsabilidades de éstos en el manejo y aprovechamiento de los recursos.

Es así como Uribe (2006, p. 13) expresa:

A comienzos de la década de los años noventa la nueva constitución dio un nuevo impulso al proceso de descentralización de la administración pública $\mathrm{y}$ consagró a la educación como un derecho de la persona y un servicio público con función social.

En esta dinámica de evolución y de políticas de los diferentes ministerios por las necesidades de urgencia Uribe (2006, p. 1) expresa que "El desarrollo de la educación en Colombia a lo largo de la primera mitad el siglo XX fue lento: A finales de los años cuarenta el país tuvo un bajo nivel de escolaridad". Datos como estos ratifican la necesidad de retomar en el país temas como: niveles de escolaridad, oportunidad para todos, igualdad, equidad y la educación como un derecho de toda persona.

Además, Uribe (2006, p. 2) menciona algunos factores que en Colombia han sido motivo de agenda en el sistema educativo desde años atrás y que aún prevalecen, como son:

La falta de maestros preparados, la forma como se descentralizó el sistema educativo, la poca prioridad que le dieron los diferentes gobiernos a la educación y la falta de recursos fueron los obstáculos más grandes que enfrentó el sector durante la primera mitad del siglo XX.

En la actualidad el tema de los partidos políticos, las rivalidades y la polarización (como a la fecha 2019) han desfavorecido las dinámicas sociales, porque no dejan que temas como la educación generen el impacto que como aporte en el desarrollo económico, industrial y cultural es necesario. En los siglos XIX y principio de los XX las disputas por el poder y la inicial cultura de la corrupción, desde el mismo gobierno, deja legados de 
atraso y retroceso que en pleno siglo XXI afectan la dinámica social en cada región. La ley 115 del 1994 vigente a la fecha con su propuesta fiscal en el manejo de recursos, promueve la adecuada distribución y aprovechamiento de los capitales para educación y salud con la idea de beneficiar a las personas y mantener la institucionalidad.

Consecuentemente, la educación en Colombia ha sido tema de debate a lo largo de toda su historia y se mantiene un claro interés en su mejoramiento pese a las dificultades y a resultados no tan favorables. Al respecto Araya (2007) expresa que la educación en Colombia ha retomado el camino para que los hombres encuentren en ella su identidad así como una mejor forma de vivir y de proyectarse dentro de la sociedad sin dejar de reconocerse y reconocer al otro en su diferencia.

En otras palabras, se ratifica la importancia y relevancia de la educación en Colombia como componente del desarrollo nacional y se constata el protagonismo de ésta a nivel de los procesos importantes para el país. Además, se advierte que desde la educación se centran propósitos claros de calidad y oportunidad para las personas, tal es el caso del Plan Nacional de Desarrollo (PND) 2014-2018, el cual se centra en el mejoramiento del desempeño de los estudiantes y en la reducción de las brechas de inequidad, variables que son persistentes en la sociedad colombiana pese a los resultados reales de la educación y su calidad actual.

Al respecto Schleider (2016) indica que "La educación es uno de los tres pilares fundamentales del Plan Nacional de Desarrollo (PND) 2014-2018". (p. 38))

En este sentido, Colombia permanentemente mantiene su interés $y$ voluntad en proponer estrategias y recursos que consigan dar cumplimiento a los requerimientos de las sociedades actuales, para lograr así que la educación alcance los niveles de exigencia propios de la época.

Fenómenos como la fluctuación y los cambios acelerados de las sociedades actuales, indistintamente del territorio y su ubicación, exigen de la educación, como proceso que ayuda al crecimiento social, económico y cultural de los pueblos y que además impacta el desarrollo y la calidad de vida de las personas, la toma de decisiones hacia la efectividad y la eficiencia en la prestación del servicio educativo, lo cual genera inquietud ya que se trata de proponer nuevas formas de hacer las cosas.

De esta manera y con el ánimo de mejorar procesos y condiciones educativas en Colombia, la Ley 115 de 1994 propone la autonomía escolar para dar cumplimiento a lo dispuesto en la constitución de Colombia del 1991. Esta ley plantea el término de identidad a las instituciones del estado mediante los Proyectos Educativos Institucionales (PEI). Al respecto Calvo (1996) expone que desde el decreto 1860 de 1994 (decreto que regula la ley 115), se define como todo establecimiento educativo debe, con la participación de la comunidad educativa, elaborar y poner en práctica el proyecto educativo institucional.

Este decreto además expresa la forma como se decide alcanzar los fines de la educación colombiana. Todo según las condiciones sociales, económicas y culturales de su medio, lo cual le da un toque de identidad y distinción dentro del conjunto de establecimientos educativos: es decir, las instituciones se distinguen unas de las otras mediante el proyecto educativo institucional.

Asimismo, el Decreto 1860 que reglamenta la Ley 115, plantea una propuesta específica y orienta la construcción del PEI con la participación de los miembros de la comunidad educativa, de igual manera cuida que sea evidente el cumplimiento de los fines 
de la educación y que éstos velen por el fortalecimiento y mejoramiento social del entorno, gestado éste desde su propia comunidad.

Para tal tema Valderrama (2008) agrega:

Mediante la ley de competencias y recursos del 93 y la ley general de educación del 94 refrendada por la ley 715 del 2001 los establecimientos educativos adquirieron autonomía para elaborar sus PEI y con él sus planes de estudio, métodos pedagógicos y la manera de organizar su trabajo. (2008, p.13).

De esta manera, en los últimos años el PEI llega a ser parte de la dinámica de mejoramiento de la educación colombiana que se propone, con el fin de contextualizar el proceso educativo de cada entorno en particular.

Araya $(2007$, p. 32) en acuerdo con las bondades del PEI habla en favor de las dinámicas evolutivas $\mathrm{y}$ las propuestas educativas de descentralización y autonomía institucional: "La reforma se hace posible y se facilita gracias a un nuevo marco institucional, que combina criterios de descentralización y competencia por recursos, con razones de discriminación positiva y de acción proactiva del estado".

Con respecto a cómo debe ser adoptado el PEI en cada una de las instituciones educativas, Araya (2007, p. 48) expresa "que es un instrumento político y técnico que orienta el quehacer del establecimiento escolar y de sus diferentes actores, que explica la propuesta educacional y especifica los medios que se pondrán en marcha para realizarla"

Es por esto que el PEI debe ser considerado por cada uno de los miembros de la comunidad educativa de cada institución como una herramienta potente para re significar la presencia de la escuela en cada contexto.

Valderrama (2008, p. 15) hace alusión a lo característico del PEI en cada EE al expresar: "Instituida por la Ley General de Educación, la autonomía escolar brindó a cada establecimiento educativo la facultad para definir su identidad y plasmarla en su Proyecto Educativo Institucional".

En concordancia con el aporte del autor antes mencionado, el PEI, en cada EE deberá reflejar qué tipo de institución formadora es, cuál es su propósito y la oferta educativa que ofrece; además deberá identificar el contexto social, cultural donde se encuentra instalada (sin desconocer que contexto también es región, departamento, país). Mientras que la realización de su seguimiento y control estará en las manos de los responsables directos: docentes, directivos y comunidad educativa en general.

El PEI contiene dos propósitos: político y técnico. Lo político alude a la participación en su construcción de los actores del proceso educativo y lo técnico a los contenidos de las políticas, metas y acciones en cada uno de sus componentes, lo cual dibuja la vigencia de los PEI y su intención de mejoramiento $\mathrm{y}$ transformación educativa mediante procesos de descentralización y autonomía de la institucionalidad en Colombia. Este recurso apunta a la transformación de las instituciones, le otorga el protagonismo, responsabilidad y acción comunitaria a los establecimientos educativos en cada una de las regiones del país.

Valderrama (2008, p. 14) expone:

La descentralización política y
administrativa colombiana a
partir de la nueva constitución
política del 91 ha rido
fundamental
posicionamiento hacia r el


establecimientos educativos (EE) como espacios donde se materializa la educación pertinente y de calidad para todos, a través de proyectos concretos como el PEI, definidos y concertados por toda la comunidad educativa.

Schleicher (2016, p. 145) hace alusión a algunos elementos contentivos en el PEI que ratifican la intención de identidad y autonomía y que hacen parte de los componentes de este proyecto: "De acuerdo con la Ley General de Educación de 1994, todos los centros educativos en Colombia tienen el derecho de definir sus propios currículos y planes de estudio mediante sus PEI.".

Como el currículo y en éste los planes de estudio, el PEI también contiene en su estructura un modelo pedagógico que es adoptado por la comunidad educativa y que soporta la propuesta formativa y curricular de la institución educativa, (tanto el modelo como el currículo se encuentran en el componente pedagógico), un componente teleológico que contempla los valores, perfiles, la visión y la misión, ideales que se pretende conseguir en el estudiante y en cada uno de los miembros de la comunidad educativa.

De igual manera un componente administrativo y otro comunitario donde el primero atiende las orientaciones de cómo administrar bien los recursos económicos y de gestión en la institución, y el segundo cómo orientar la comunidad de personas a cargo y cómo se proyecta la institución hacia la comunidad en general.

De todo este conjunto de partes y componentes del PEI son conocedoras las instituciones educativas, pero esto no ha sido suficiente para garantizar su efectividad. El ministerio y las Secretarias de Educación (SE), no han realizado el seguimiento y control institucional de los PEI, lo que provoca que se desvíe su propósito y con ello la oportunidad de ser el proyecto educativo que funja, para cada institución, como instrumento guía hacia procesos de calidad institucional y educativa.

En función de lo antes expuesto, se puede decir, que a pesar de que la intencionalidad del PEI está bien delimitada la realidad no ha sido tan positiva, ya que en la mayoría de los docentes se ha desvirtuado en el tema de la autonomía y se ha caído en el facilismo y la falta de reflexión al punto de hacer las cosas de manera improvisada, sin tener en cuenta procesos de construcción y transformación; lo que da pie a considerarla como una autonomía amañada y mal enfocada.

Al respecto, Calvo (1996), plantea que el tema de la promulgación del PEI de manera abrupta, no ha sido lo único confuso. Expresa el autor, que éste nunca logró permear la labor docente en su totalidad, es decir, los PEI tomaron a los docentes por sorpresa, sin estar motivados y capacitados para afrontarlos lo cual creó, por lo general, confusión, desánimo o rechazo"

Otro de los fenómenos adversos que giraron en torno a su elaboración en principio fue la falta de orientación, la incertidumbre en cuanto al impacto que generaría en sus prácticas y el afán de cumplir con éste de acuerdo con las expectativas desde la norma. En atención a lo anterior Araya (2007, p. 30) expone "La Reforma Educacional se caracteriza por ser gradual, incremental y posibilitar el desarrollo de Proyectos Educativos Institucionales propios en cada escuela y liceo", es decir, que las reformas y propuestas no deben ser impuestas ni esperar que su acogida sea de manera inmediata.

Para Lucio (1995, c.p. Calvo 1996), las acciones remediales al rechazo y la falta de conocimiento tuvo su momento de atención por parte del Estado: como respuesta a las 
actitudes de rechazo del PEI, rápidamente el Ministerio ofreció apoyo y, junto con la asesoría ministerial, las ofertas de expertos, seminarios, textos y manuales, producidos por autores individuales, colegios privados y editoriales inundaron el mercado.

Es así como, pese a que al inicio de la propuesta del PEI se realizan acciones de acompañamiento y capacitación, a la fecha es evidente que existen, en las instituciones educativas, diversas formas, estilos $y$ propósitos de concebir el PEI. Es común encontrar copias del PEI, muchas de ellas archivadas sin ninguna funcionalidad y otras sin alcanzar protagonismo en el sistema educativo.

No obstante, García y col. (2012, p. 36), reconocen todos los atributos y bondades que encierra el PEI y expresan, "El PEI contribuye a medir los logros y la ejecución de las actividades, involucra $\mathrm{y}$ da sentido de pertenencia".

Sin embargo, la realidad es otra, los docentes y la institución misma consideran el PEI como una norma para cumplimiento de las directrices y políticas, lo que aleja al PEI de ser el instrumento que da pertenencia a sus actores y le genera pérdida de vigencia, oportunidad y efectividad en la intención de mejoramiento de los establecimientos educativos.

Dentro de los procesos de formación y empoderamiento Calvo (1996) enfatiza en el PEI cuando da a entender que éste exige un proceso de progresivos logros y acuerdos. Sólo mediante su conocimiento, además del reconocimiento de su proceso será posible tomar medidas que incidan en el mejoramiento de la calidad de la educación.

El planteamiento del autor antes mencionado reitera la importancia de que el PEI no sea mera norma o sólo un instrumento de consulta esporádico. En los procesos de eficiencia escolar y calidad educativa, el PEI está concebido como instrumento de orientación permanente de cada una de las acciones de los miembros de la comunidad educativa donde los docentes serán siempre los primeros convocados.

La apreciación de Calvo (1996) es el reflejo actual del PEI. Desde sus inicios y a la fecha el PEI genera en los docentes y al interior de las instituciones educativas sentimientos contradictorios. La propuesta del proyecto confunde a los actores educativos sobre el gran compromiso de su elaboración y la posibilidad de construir un ideal a partir del accionar de los docentes y demás miembros de la comunidad educativa.

En este orden de ideas, Calvo (1996) se adentra un poco más sobre el tema y habla de uno de los actores importantes en educación desde la ley: Se refiere a la Ley General de Educación que concibe la formación del maestro colombiano como un profesional, con un perfil de formación para el ejercicio de la autonomía intelectual en una cultura pedagógica, universal y nacional (MEN, 1995, p. 12). Se puede notar entonces como en Colombia, desde la misma ley las instituciones formadoras de docentes están llamadas a formar con autonomía intelectual, donde la autoconstrucción y la autocrítica son componentes que aportan para que los docentes sean profesionales con capacidades académicas y profesionales suficientes para atender las exigencias y requerimientos de la educación que dirigen.

Calvo (1996) hace hincapié en el tema de ser docente en Colombia: La ley General de educación insiste que el maestro, más que un funcionario, es un analista de los procesos de formación, aprendizaje, educación y enseñanza, es decir, un profesional integro con capacidad de autoconstruirse y en formación constante en atención a las políticas y las sugerencias de la educación. De esta manera se compromete al docente a 
mantener una actitud de cambio, de reflexión constante y de toma de decisiones asertivas frente al reto de la calidad.

De esta manera, si el desempeño del rol de los docentes (prácticas pedagógicas) está desarticulado y desligado de las orientaciones propias de la institución contempladas en el PEI, no se puede hablar de identidad $y$ autonomía, que es finalmente lo que se sustenta en este proyecto.

Esta situación de desacato, indiferencia y falta de compromiso por parte de docentes, directivos y comunidad educativa en genera, pone en riesgo la efectividad y el logro de los propósitos de formación de un ser íntegro con capacidades académicas óptimas (como lo contempla la ley desde la constitución política colombiana), y deja al establecimiento educativo en situación de vulnerabilidad en los procesos y en el propósito de calidad institucional. Además, le quita a la institución la posibilidad de ser una oportunidad de mejora de la calidad de vida de las personas que pertenecen a ese contexto.

Asimismo, a todo lo antes mencionado se puede sumar la falta de identidad $y$ coherencia entre las políticas y las acciones educativas, lo cual coloca a la escuela en posición de fragilidad en sus propuestas de formación ya que de no lograr establecer una dinámica plenamente identificada, no se conoce a ciencia cierta cómo hacer las cosas lo que conlleva a un desorden a nivel académico por la existencia de una variedad de prácticas, sin identidad ni coherencia, dentro de la institución.

En efecto, el PEI beneficia a las instituciones ya que no sólo contempla las políticas institucionales propias de cada establecimiento educativo, sino que es a la vez orientador en cuanto a las formas y maneras de proceder de cada uno de sus miembros; además, es fiel a la atención de las necesidades que cada contexto identifica y apunta para su mejoramiento, por ende persigue que las prácticas pedagógicas apunten a los propósitos en el establecidos los cuales se centran en las necesidades de la sociedad para la cual fue construido.

Sin embargo, la resistencia existente por parte de los miembros del sistema educacional convierte al PEI, en solo una norma en papel, y lo hacen estar ausente de los procesos de formación y mejoramiento institucional. En este sentido, Araya (2007) incentiva a la comunidad educativa a hacer uso de su autonomía y al mismo tiempo reconoce la necesidad de un proyecto que oriente y que distinga las metas hacia las cuales dirigirse, el cual identifica con el proyecto educativo institucional PEI.

El PEI es de esta manera, el telón desde donde se proyecta todo accionar que en sus inicios es creado hacia la necesidad, metas y aspiraciones de toda una comunidad que de manera crítica y prospectiva mira a futuro los logros de su institución educativa y la oportunidad de desarrollo para su comunidad.

Angarita (1995, c.p. García y col. 2012) relaciona lo postulado por García y otros:
Desde la parte legal el Proyecto Educativo es considerado como un instrumento que prevé y organiza acciones administrativas, de planeación, técnico-pedagógicas y otras, para resolver necesidades $y$ expectativas de la comunidad educativa, enmarcadas en las políticas y disposiciones legales nacionales y regionales (p. 34).

En este mismo orden de ideas, todos los autores antes mencionados ratifican la legalidad de la cual goza el proyecto PEI, lo cual le convierte en una herramienta de planeación. De ahí que su construcción debe 
realizarse con y para la comunidad educativa, a fin de que refleje realmente las necesidades $\mathrm{y}$ requerimientos de su entorno lo que realmente le convierte, en atención a sus directrices, en un referente para la toma de decisiones, así como guía de las acciones pedagógicas de docentes.

En consecuencia, es razonable entender que la reforma que se propone a partir del PEI como instrumento identificador de las instituciones del estado, no pretende el cambio de la institución sino que desde este instrumento, el PEI, se potencialicen varios de sus aspectos: currículo, prácticas pedagógicas, consolidación de una comunidad educativa, procesos identificadores como evaluación, formación integral hacia la consecución de la calidad y la re significación de la institucionalidad; buscando con ello mayor protagonismo institucional y una mejor identidad entre el contexto y el servicio educativo.

Un aspecto importante que rodea la propuesta del proyecto PEI son las dinámicas sociales. Tanto las leyes como los ideales son cambiantes, no se construyen para quedarse, se construyen, pero se mantienen en actitud expectante para hacer adecuaciones, cambios $\mathrm{y}$ reformas que apunten a la efectividad y eficiencia de acuerdo con las realidades del momento. De igual manera, la educación es un proceso favorable a las necesidades $y$ requerimientos de una sociedad en constante evolución. El PEI llega con la intención de aportar en aras de potenciar y re significar cada entorno, desde los colegios y escuelas que le pertenecen.

En este sentido, Valderrama (2008) Valora, a partir del PEI, la presencia de los establecimientos dentro de las comunidades al expresar: "El establecimiento educativo no está aislado, sino que se enmarca en un contexto que se transforma continuamente" (p.39).
En efecto, la primera tarea de un establecimiento educativo es reconocerse miembro de una comunidad y prestar el servicio de acuerdo a las características y necesidades de sus beneficiarios (estudiantes, familias). Se hace necesario un esfuerzo para conocer muy bien las características de los estudiantes y sus familias, así como sus necesidades con el fin de apuntar de manera efectiva a los procesos de calidad y transformación necesarios para el contexto al cual se pertenece.

Pese a toda esta intencionalidad pedagógica y de reforma en pro de mejorar la acción educativa colombiana, la realidad es otra. Las instituciones educativas han caído en un estado de inercia y permisividad que no les permite cuestionar las formas de accionar de los docentes. Las instituciones subestiman y relegan las potencialidades del PEI como instrumento que guía, orienta y mediante el cual también se puede ejercer control, el PEI revisa la realidad y la confronta con lo que en él se plantea, es decir, el PEI puede ser utilizado tanto como recopilación de la realidad contextual y el ideal de formación, como de revelador de su coherencia e identidad institucional.

En atención a este fenómeno lo ideal sería lo que propone García y otros. (2012):
La puesta en marcha de mecanismos de seguimiento $y$ control dará a cada institución, un claro panorama que evitará las improvisaciones de sus miembros conducirá a saber si lo que se hace responde a las necesidades de la población que se desea formar y si esto se está logrando (p. 36).

García y col. (2012) continúan su sustentación diciendo: "Al declarar el PEI como referente de la práctica pedagógica, 
resulta obligatorio revisar tópicos tales como: los aspectos considerados relevantes en él, la participación de la comunidad académica en su construcción y la divulgación del mismo" (p. 34). Los aspectos mencionados por los autores son importantes para la instrumentación del proyecto PEI ya que si la institución no cuenta con un plan de revisión, reflexión y mantenimiento de éste de cara a la realidad, todo lo que allí se contempla pierde vigencia y se corre el riesgo de ser olvidado y que la multiplicidad de prácticas y maneras de hacer las cosas sin identidad alguna se convierta en la cultura institucional, a pesar de que se cuente con un proyecto educativo.

En efecto, una institución muestra solidez una vez que cuenta con procesos $\mathrm{y}$ mecanismos de seguimiento y evaluación periódica que involucra a toda una comunidad educativa, en el caso del PEI, esto le permite estar vigente, con validez de manera permanente y con vigencia en el tiempo. Al respecto, Araya (2007) expresa: "Si esta tarea de evaluabilidad no logra realizarse, lo que expresa el PEI sufre la amenaza de perder vigencia y sentido para la comunidad educativa" (p.50).

En este mismo orden de ideas y a fin de afianzar aún más la idea puntual de lo que representa el PEI para una comunidad en cuanto a vigencia y evaluabilidad, Araya (2007) expresa: "Lo que se solicita para la evaluabilidad y vigencia del PEI, es que la comunidad se ordene de tal modo, que exista la posibilidad de revisar el documento que expresa sus orientaciones y sueños mayores" (p. 50). De allí que es muy claro que para la expansión y permeabilidad que deben tener los PEI en cada institución deben existir autores y responsables de su elaboración.

$\mathrm{Al}$ respecto, Calvo (1996) haciendo alusión a las propuestas del PEI (desde el MEN en 1994) aclara que sin excusas (aún para aquellos procesos más avanzados) el hecho de que los Proyectos Educativos Institucionales son una construcción colectiva por la comunidad educativa, compromete la intencionalidad de cada uno de los miembros: Maestros, alumnos, padres, la localidad en la cual se encuentra la escuela, y más aún: que lo ideal es que el PEI participe de los planes de desarrollo municipales PDM, plan educativo municipal PEM y planes de desarrollo regionales (esto lo empodera aún más y lo hace más efectivo y válido una vez se lleve a cabo).

La propuesta ideal del PEI como instrumento orientador con identidad propia para cada institución, es que sea construido por la comunidad educativa, es decir por las personas que le son propias del contexto, que cuiden su funcionabilidad y se inmiscuyan $y$ comprometan a tal grado que estén expectantes de lo que sucede.

En este sentido, Araya (2007) aclara: "La propuesta educacional señalada en el PEI es obra de personas, y como tal, es perfectible, flexible y dinámica" (p. 50).

De igual manera, Araya (2007) se refiere a la participación y el compromiso de la comunidad educativa, a quien reconoce como atores principales en la construcción del PEI, expresa: "El Proyecto de Ley General de Educación, consolida como uno de sus principios fundamentales el reconocimiento, la valoración y la participación de todas y todos los miembros que componen una comunidad escolar" (p. 40). El autor deja claro que es desde la iniciativa de estos miembros que nace la promulgación del proyecto institucional y desde donde no sólo se gestan la misión, la visión y el ideal formativo, sino que también son los veedores de su cumplimiento.

Es así como el PEI no es solo un instrumento, es una unidad de comunidad y de consenso por un bien común, desde el ministerio como ente dinamizador y veedor 
de los procesos de calidad en las instituciones educativas. Al respecto, Valderrama (2008) conceptualiza la comunidad educativa desde la intencionalidad y el papel que cumple desde la ley:

Comunidad educativa: en
concordancia con el artículo $6^{\circ}$ de la
Ley General de Educación y el
artículo 18 del Decreto 1860 de
1994, la comunidad educativa está
conformada por las personas que
tienen responsabilidades directas
en la organización, desarrollo y
evaluación del proyecto educativo
institucional que se ejecuta en un
establecimiento educativo (p. 149).

Quiere decir que por ley la comunidad educativa está plenamente identificada y es responsable dentro de los procesos de mejoramiento, en ser parte de la construcción (y por ende del seguimiento y veeduría) de su PEI.

En efecto, es en las comunidades educativas de cada institución donde se encuentran inmersos y activos los docentes como actores y gestores en la puesta en marcha de lo propuesto en su PEI (al menos eso es lo que la norma contempla), son las prácticas pedagógicas de los docentes las que direccionan y encausan el rumbo de los ideales institucionales en cuanto a los logros académicos de los alumnos y por ende el logro de la calidad educativa en cada institución (que es finalmente lo que se pretende con la construcción y puesta en marcha del PEI).

Asimismo, Bellei y col (2013, p. 109) mencionan: "Tanto los estudios sobre los logros académicos de los alumnos como los de mejoramiento de los sistemas educacionales coinciden en señalar que la calidad del cuerpo docente es el factor clave de la calidad educacional".
En efecto, esto es ratificado desde las guías del MEN (1995) referidas por Calvo (1996) cuando dice que contempla como vital y necesario el empoderamiento de los miembros de la comunidad educativa, en especial el de los docentes en los procesos e intencionalidad de cada estrategia. En este sentido, la estrategia de formar docentes vinculados a los procesos de formulación del PEI, de su puesta en marcha y de su seguimiento lo cual depende, tal como lo exigen los nuevos lineamientos, de una muy buena formación y disposición del docente quien debe estar en actitud de logro de solidez en lo pedagógico, lo disciplinario, lo axiológico y lo ciudadano.

El llamado a la integralidad del docente en la actualidad no admite actitudes como la indiferencia y resistencia a la transformación. Actualmente las instituciones formadoras de maestros están llamadas a generar en sus egresados actitudes y aptitudes que los hagan profesionales con dominios y habilidades desde lo disciplinar y mejor aún desde lo ético, lo humano y lo pedagógico, capaces de ser autocríticos, reflexivos y de continuar procesos de autoformación.

Al respecto, Denegri y col. (1996, p. 8) asumen igual postura que Calvo (1996) al expresar: "Para alcanzar un alumno con las particularidades definidas, se requieren docentes que cumplan un perfil coherente con los objetivos planteados por el establecimiento en el PEI". Se infiere que el tema del perfil del docente es determinante para llevar a cabo o no lo estipulado en el PEI.

Con respecto al resultado del compromiso que adquiere el docente en los procesos institucionales, al reconocerse como agente garante de llevar a cabo las políticas descritas en el PEI, Flores y col. (2013, c.p. Montes (2013) opinan: El rol del docente, desde la teoría, propone un diálogo permanente en el ejercicio práctico de su 
enseñanza y de su práctica pedagógica, este accionar, es el escenario perfecto para discernir sobre la calidad de la educación. La reflexión y diálogo interno sobre las prácticas pedagógicas, la calidad en la instrucción y el impacto positivo o negativo en los procesos de los estudiantes es justo y necesario mirarlos, en cada establecimiento educativo, desde el rol y visión del docente.

En efecto, autores como Hernández y col. (2001) apoyan los diálogos entre docentes cuando aclaran que las observaciones que puedan hacerse en el proceso de enseñanza son comunes y especifican que es posible identificar un número significativo de docentes y estudiantes que se enfrentan críticamente, para apoyar o estar en oposición, cuando se requiere encontrar sentido a lo que habitualmente hacen en sus aulas.

En consecuencia, la posición crítica de docentes y estudiantes frente a la realidad permite encontrar las razones de los resultados que se obtienen, al igual que encontrar la manera de mejorar y revertir en favorables las situaciones adversas. Es posible decir entonces que las aulas son escenarios donde se constatan las eventualidades favorables oportunas o no. El docente es quien valora, incentiva $\mathrm{y}$ hace de estos espacios una oportunidad, o por el contrario es también quien mediante una posición radical, ajena a su esencia como docente, pierde la oportunidad de mejorar su práctica y el desempeño de sus estudiantes.

Será entonces prioritario hacer esfuerzos para que los docentes tengan oportunidad para el cambio. Schleicher (2016) habla en tono esperanzador cuando dice: "En los últimos años se han realizado mayores esfuerzos para mejorar la disponibilidad y la calidad de las actividades de desarrollo profesional" (p. 158). Es así como mediante cursos, capacitaciones y actualizaciones se espera trascender la formación tradicional sin que por ello se deje de revisar lo que realmente sucede con los docentes en las aulas cuando se detectan, a nivel general, falencias y desfases en lo que realmente intenta lograr la educación: ser la protagonista en la transformación y el desempeño eficaz de las personas.

Es aquí donde, por contener el PEI todo un ideal y un direccionamiento de cómo lograrlo en la comunidad para la cual fue elaborado, es posible combinarlo con las prácticas pedagógicas de cada institución educativa y convertirlo en plataforma desde la cual existe la posibilidad de lograr la calidad en los procesos educativos de cada EE.

De igual manera, una vez llevado a cabo, el PEI tiene validez, pero son los docentes quienes lo materializan, son ellos, los docentes, los de mayor impacto en lo plasmado en el proyecto educativo, pero es necesario que se identifiquen con el proyecto y participen efectivamente en su construcción, lo contrario es dar paso a instituciones con variedad de formas de enseñar, sin identidad $\mathrm{y}$ sin consonancia institucional lo que provoca al interior de las mismas aislamiento e indiferencia por el consenso.

En efecto, no es osado pensar que la calidad de la enseñanza tenga su asidero en las prácticas pedagógicas (a pesar de que hay factores externos a la escuela que también tiene que ver con los resultados obtenidos con los estudiantes). Son las prácticas pedagógicas las que finalmente luchan por conseguir resultados en los estudiantes. Con esa intención se construye el PEI, con el fin de ser la carta de navegación de toda una comunidad educativa, en especial de los docentes que permanecen dentro de las aulas en constante contacto con los estudiantes y siguiendo derroteros como: Los planes de 
estudio, el sistema de evaluación institucional, llevar a cabo un modelo pedagógico. Son los docentes quienes no solo deben tener pleno conocimiento del PEI, sino que deben estar convencidos, conscientes de que en el proyecto educativo PEI se encuentra la forma de hacer las cosas bien.

En este sentido, la labor del docente, como bien se ha dicho es integral, su praxis, sus acciones, su formación tiene componentes variados que hacen de esta una profesión integral, no basta con conocer del tema o la disciplina que se orienta, se debe además saber de pedagogía, de método, de didáctica, de leyes, de reformas, de políticas educativas entre otras, es decir, no solo se debe saber lo disciplinar, se trata de autoformarse, ser flexibles y autocríticos; todos estos elemento, hoy por hoy, se suman al reto de ser docentes.

\section{REFLEXIÓN FINAL}

En consecuencia y a manera de reflexión final hay que recordar que ya no se habla de la profesión docente como la profesión por la que se opta al momento de no tener otra opción, sino que ahora es un reto, es una profesión con elementos variados $\mathrm{y}$ complejos, con profesionales que no siempre reúnen lo requerido académicamente, sin embargo, tienen en sus manos el futuro de las personas, de las comunidades e incluso de naciones enteras.

Todo lo antes expuesto justifica la necesidad de reformas y propuestas en educación como en efecto lo es el PEI, donde para cada establecimiento educativo la autonomía y la identidad son una oportunidad de ser asertivos y al mismo tiempo retan al docente a que se trasforme, a que plasme en el PEI de manera anticipada lo que quiere lograr, y a conocer cómo hacer lo que en el PEI se contempla.

Pensar que docentes e instituciones deciden la manera como llevar a cabo la educación en determinado contexto, es estar arraigados al pasado, todo ha cambiado en la actualidad. La concesión de autonomía apunta a que contrario al autoritarismo, el facilismo y la falta de actualizaciones, tanto los docentes como las instituciones se piensen, se proyecten y apuesten al logro de nuevas metas acordes con las exigencias de un mundo que no cesa de crecer y de evolucionar.

Educar ahora es lograr transformación y desarrollo, es pensarse dentro de un contexto, observarlo, detectar lo que éste requiere de las personas y garantizar que la escuela lo puede suplir mediante la educación que imparte. Así está pensada la educación actual, una formación propia, con una identidad plena para las personas que en este momento se educan.

En cuanto a la educación colombiana, la exigencia actual es que se contemple en el PEI el ideal que se persigue y que reclama la comunidad; es así mismo vivenciar un modelo educativo acorde a las nuevas exigencias, es conocer a cabalidad la manera, los recursos y las formas de cómo lograrlo. El PEI lleva la responsabilidad de ese gran ideal.

Acciones como la reflexión permanente del docente, la evaluación institucional (reglamentada como requisito para ser realizado anualmente en cada $\mathrm{EE}$ ), la escritura crítica de los maestros y la revisión del impacto educativo en cada establecimiento de formación son elementos dinámicos en una constante de mejoramiento. Ser indiferentes a esa realidad, mantenerse al margen de nuevas propuestas y políticas educativas, cierra las posibilidades de evolución a las instituciones y a los profesionales de la educación.

La nueva era, llamada la aldea global, en la que toda la sociedad está inmersa, no permite detenerse, no se queda estática en espera que los procesos evolucionen por si solos. 
Ha llegado la época en la que la profesión docente, que es una de las más completas, exigentes y retadoras, alce la voz y deje claro que el ideal de sociedad que se persigue sólo es posible alcanzarlo mediante la evolución de la educación.

El PEI junto a la voluntad y buena disposición de los docentes puede convertirse en un instrumento político en cada establecimiento educativo, puede llegar a ser ideología, un tipo de política inquebrantable desde donde se aborde un sueño formativo, se revise constantemente y se evalúe, hasta que finalmente se refleje en egresados que aporten de manera diferente a la dinámica social actual.

En efecto, la solución no está en unas pocas manos, pero el cambio de un colectivo se inicia de manera individual y prosigue hasta conseguir masas. Hay esperanzas que muy pronto llegue esa voluntad, de que Colombia sea modelo para otros en materia de educación, que las voces lleguen lejos y se pueda ver de cerca el éxito, que una acertada motivación impulse a experimentar cosas nuevas, a atender directrices productivas, que faciliten y garanticen a la comunidad educativa en general llegar a ser artífices del cambio que Colombia está esperando para sumarse a los países con mejores resultados; donde se consigan personas más felices, capaces, autónomas, competentes y propositivas como lo requiere el mundo laboral y social del momento.

\section{REFERENCIAS}

Araya, E. (2007). Asistentes de la Educación en la Reforma Educativa Ministerio de Educación Nacional. Colombia: Ministerio de Educación República de Chile: Red Académica.

ttp://revistas.pedagogica.edu.co/index.ph p/RCE/article/view/5400"

Bellei, C., Poblete, S., Sepúlveda, P., Orellana, V., Abarca, G. (2013). Situación Educativa de América Latina y el Caribe: Hacia la educación de calidad para todos al 2015. Oficina Regional de Educación para América Latina y el Caribe COREALC/UNESCO DOCENTES Revista Colombiana de Educación Santiago de Chile: Red Académica. http://revistas.pedagogica.edu.co/index.p $\mathrm{hp} / \mathrm{RCE} /$ article/view/5400\&quot;

Calvo, G. (1996). Los proyectos educativos institucionales y la formación de docentes. Revista Colombiana de Educación, (33)

Denegri, M., Aguayo, C., Vargas A. (1996) El Proyecto Educativo Institucional como instrumento de reflexión y participación de la Comunidad Educativa.

García A, O. González P., A. y Sierra F., R. (2012). Proyecto educativo institucional y la práctica pedagógica en las Instituciones de Educación Superior (IES): una relación imaginaria. Justicia juris, 8(1), 30-39 ISSN 1692-8571, Vol. 8. № 1. Enero - Junio 2012 Pág. 30-39. Atlántico: http://www.scielo.org.co/scielo.php?scrip $\mathrm{t}=$ sci_serial\&pid=1692-

8571\&lng=es\&nrm=is"

Hernández Pérez, J; Ramírez Martínez, S. y Souto González, X.M. (2001) Evaluación y aprendizaje. Una propuesta para mejorar el rendimiento escolar. Valencia: Nau Llibres. http://www.ub.edu/geocrit/b3w357.htm

Ministerio de Educación Nacional (1994). Ley General de Educación 115. República de Colombia

Ministerio de educación nacional (1995). Guías

Montes, M., (2013). La formación docente en el marco de la política de calidad de la Educación Básica en Colombia. Revista ESPACIOS. Vol. 38 (№ 20). Pág. 26

Schleicher, A. (2016). Revisión de políticas nacionales de educación La educación en Colombia Ministerio de Educación Nacional para esta versión en español París: OCDE. https://www.mineducacion.gov.co/1759/ articles-356787_recurso_1.pdf 
UNESCO (2017). Guía para asegurar la inclusión y la equidad en la educación.

Disponible en:

https://unesdoc.unesco.org/ark:/48223/ pf0000259592

UNESCO (2000). EDUCACION PARA TODOS. FORO MUNDIAL SOBRE LA EDUCACION. Disponible en:

https://unesdoc.unesco.org/ark:/48223/ pf0000121117_spa

Uribe, J. (2006). Evolución de la educación en Colombia durante el siglo XX Revista del
Banco de la República Volumen: LXXIX Número: 940 Bogotá, Colombia: Banco de la República. http://www.banrep.gov.co/es/node/10495 http://www.bancomundial.org/es/topic/ education/overview

Valderrama R. (2008). Guía para el mejoramiento institucional de la Autoevaluación al Plan de Mejoramiento, Guía 34. Colombia Ministerio de Educación Nacional 